\title{
Progress of Geant4 electromagnetic physics developments and applications
}

\author{
Vladimir Ivanchenko ${ }^{1,2, *}$, Alexander Bagulya ${ }^{3}$, Samer Bakr $^{4}$, Marilena Bandieramonte ${ }^{2,5}$, \\ Denis Bernard ${ }^{6}$, Marie-Claude Bordage ${ }^{7,8}$, Jeremy Brown ${ }^{9}$, Helmut Burkhardt ${ }^{2}$, Paolo \\ Dondero $^{10}$, Sabine Elles ${ }^{11}$, Vladimir Grichine ${ }^{3}$, Susanna Guatelli ${ }^{4}$, Farah Hariri ${ }^{2}$, Alexander \\ Howard $^{12}$, Sebastien Incerti ${ }^{13,14}$, Soon Yung Jun ${ }^{15}$, Omrane Kadri ${ }^{16}$, Ioanna Kyriakou ${ }^{17}$, \\ Michel Maire ${ }^{18}$, Alfonso Mantero ${ }^{10}$, Mihaly Novak ${ }^{2}$, Daren Sawkey ${ }^{19}$, Igor Semeniouk ${ }^{6}$, \\ Anton Sokolov ${ }^{20,21}$, and Laszlo Urban ${ }^{18}$ on behalf of the Geant4 Collaboration \\ ${ }^{1}$ Tomsk State University, 634050 Tomsk, Russia \\ ${ }^{2}$ CERN, CH1211, Geneva 23, Switzerland \\ ${ }^{3}$ Lebedev Physical Institute, 119991 Moscow, Russia \\ ${ }^{4}$ University of Wollongong, Center for Medical Radiation Physics, Wollongong NSW 2522, Australia \\ ${ }^{5}$ University of Pittsburgh, 4200 Fifth Avenue, Pittsburgh, PA 15260, USA \\ ${ }^{6}$ LLR, CNRS/IN2P3, Ecole Polytechnique, 91218 Palaiseau, France \\ ${ }^{7}$ CRCT, UMR 1037 INSERM, Université Paul Sabatier, Toulouse, France \\ ${ }^{8}$ UMR 1037, CRCT, Univ. Toulouse III-Paul Sabatier, F-31000 Toulouse, France \\ ${ }^{9}$ Delft University of Technology, Radiation Science and Technology, 2629JB Delft, The Netherland \\ ${ }^{10}$ SWHARD SRL, Via Greto di Cornigliano 6r, 16152 Genova, Italy \\ ${ }^{11}$ IN2P3/LAPP, 74941 Annecy-le-vieux, France \\ ${ }^{12}$ College Imperial, London, United Kingdom \\ ${ }^{13} \mathrm{CNRS}$ /IN2P3, CENBG, UMR 5797, F-33170 Gradignan, France \\ ${ }^{14}$ Université Bordeaux, CENBG, UMR 5797, F-33170 Gradignan, France \\ ${ }^{15}$ Fermi National Accelerator Laboratory, Batavia, IL, USA \\ ${ }^{16}$ King Saud University, Riyadh, Saudi Arabia \\ ${ }^{17}$ Medical Physics Laboratory, University of Ioannina Medical School, Ioannina 45110, Greece \\ ${ }^{18}$ Geant4 Associate International Ltd., United Kingdom \\ ${ }^{19}$ Varian Medical Systems, Palo Alto, CA 94304, USA \\ ${ }^{20}$ Moscow State University, Moscow, Russia \\ ${ }^{21}$ Institute of Theoretical and Experimental Physics, 117218 Moscow, Russia
}

\begin{abstract}
We report on developments of the Geant 4 electromagnetic physics sub-libraries of Geant 4 release 10.4 and beyond. Modifications are introduced to the models of photoelectric effect, bremsstrahlung, gamma conversion, single and multiple scattering. The theory-based GoudsmitSaunderson model of electron/positron multiple scattering has been recently reviewed and a new improved version, providing the most accurate results for scattering of electrons and positrons, was made available. The updated interfaces, models and configurations have already been integrated into LHC applications and may be useful for any type of simulations.
\end{abstract}

\footnotetext{
* Corresponding author: Vladimir.Ivantchenko@cern.ch
} 


\section{Introduction}

The Geant4 electromagnetic (EM) physics sub-libraries [1-2] are an important component of the Geant4 toolkit [3-5]. In this work, we report on recent progress in EM model developments and on updates of user interfaces, which are included in the Geant 4 versions 10.4 and 10.5beta. In our previous reports [6-7], earlier Geant4 versions were described.

Important modifications were made in the material, EM physics, and physics list Geant 4 sub-libraries. The goal was to make the most advanced EM theoretical models available to the groups performing LHC and other HEP experiment simulations. In Run-3 at LHC, higher statistics are expected than in Run-2. To provide adequate accuracy of simulations, EM physics models are under continuous review and the next-to-leading-order corrections are taken into account. This reduces systematic uncertainties in simulation predictions and provides simulation of second order effects which are important for very high energies (LHC and FCC programs), and in simulation of backgrounds in the dark matter searches [8]. Although focused on HEP experiments, these upgrades are at the same time useful for many other applications including those in medical physics and space science.

\section{EM model developments}

In Geant4 10.4, substantial improvements were introduced in the models of photoelectric effect, gamma conversion, multiple, and single scattering. Corrections to scattering of positrons and to sampling of displacement have been recently added to the Geant 4 default Urban model. In Geant4 10.5beta, some minor fixes were added and code clean-up was performed for these models as well as for bremsstrahlung models. Additionally a new alternative model for gamma conversion to $\mathrm{e}^{+} \mathrm{e}^{-}$pair was introduced. In what follows we describe the most important modifications of EM models.

\subsection{The Goudsmit-Saunderson multiple scattering model}

The Geant4 Goudsmit-Saunderson (GS) model for multiple Coulomb scattering of $\mathrm{e}^{ \pm}$is based on the "any-angle" multiple scattering model developed by Kawrakow and Bielajew [9]. The exact Goudsmit-Saunderson [10] angular distributions, computed by utilising the screened Rutherford differential cross section (DCS) for elastic scattering, can be represented in a compact numerical form suitable for fast run-time sampling of the corresponding angular deflections. The accuracy of the GS model is already appropriate for many HEP applications. However, starting from Geant4 version 10.4, the GS model provides an option of using a more accurate description of the scattering problem through the introduction of higher order corrections. The most important among these is the correction to the GS angular distributions, computed by using the screened Rutherford DCS, based on the more accurate Mott elastic DCS (spin effects) [11]. Besides the accurate multiple scattering angular distributions, the GS model provides the possibility to use the Lateral and Longitudinal Correlation Algorithm (LLCA) [12-13], that can produce lateral and longitudinal condensed history end-point distributions that are very close to those obtained by using the corresponding single scattering model. Moreover, the algorithm makes sure that the boundary is always crossed in the single scattering mode, which makes this stepping algorithm free from stepping artefacts.

As a result of these improvements, the Geant4 version 10.4 of the GS model can provide highly accurate $\mathrm{e}^{ \pm}$transport simulations even in the case of extreme geometrical conditions (e.g. high granularity calorimeters) or low energies, independently from the target atomic number as illustrated in Fig.1 (see more details in [7]). 


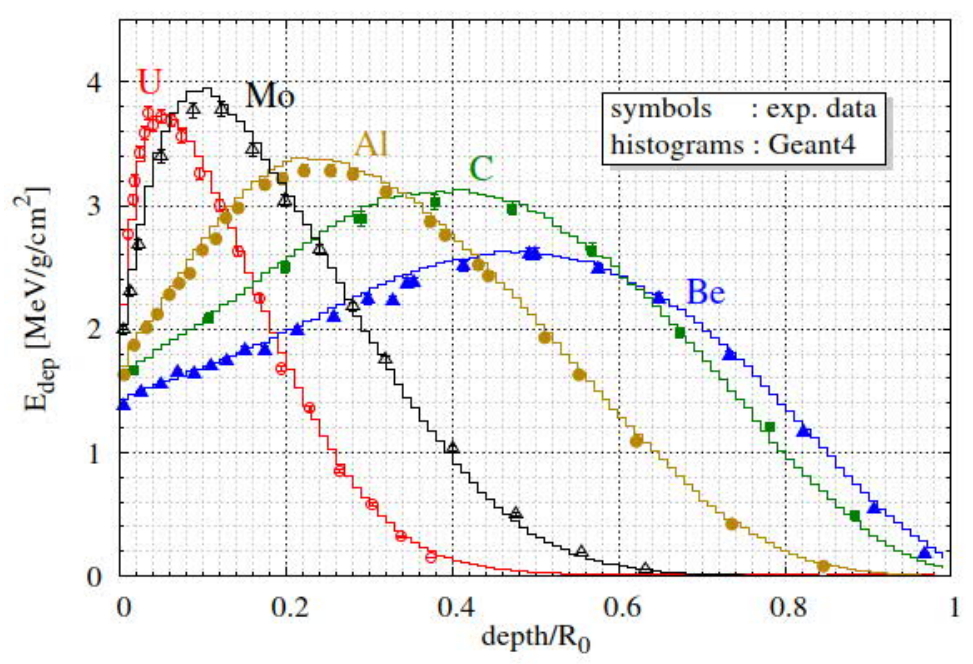

Fig. 1. Comparison of experimental (symbols [14]) and simulated (histograms Geant4 10.4. EM Opt4) electron energy deposition profiles in semi-infinite $\mathrm{U}, \mathrm{Mo}, \mathrm{Al}, \mathrm{C}$ media with primary electron energy of $1.0 \mathrm{MeV}$ and in Be with $1.033 \mathrm{MeV}$. Coordinates are normalized to the CSDA (continuous-slowingdown-approximation) range of electrons in corresponding materials.

\subsection{WentzelVI combined scattering model}

The WentzelVI multiple scattering model combined with the single elastic scattering (SS) model [15] is the current Geant4 default approach for $\mathrm{e}^{ \pm}$above $100 \mathrm{MeV}$ and for all other charged particles at any energy [5]. The limited scattering angle between multiple and single scattering is selected dynamically depending of step size and particle momentum. In Geant4 version 10.4, both models use the same Wentzel cross section [16], which does not take into account Mott corrections. For Geant4 10.5beta the SS model has been updated for $\mathrm{e}^{ \pm}$to use an alternative single scattering model which takes into account the Mott corrections. The model was developed for the $\mathrm{e}^{ \pm}$non-ionizing energy loss computations [6]. Both GS and SS models implement Mott corrections now and the results of the simulations of scattering off thin foils become very similar for these models.

\subsection{D gamma conversion to $\mathrm{e}^{+} \mathrm{e}^{-}$pair model}

The differential cross section of the conversion of a $\gamma$ ray to an $\mathrm{e}^{+} \mathrm{e}^{-}$pair in the field of the nucleus ("nuclear conversion") or of an electron ("triplet conversion") of an atom was first computed by Bethe and Heitler [17]. The phase space consists of five variables that can be chosen to be the azimuthal and the polar angles of the electron and of the positron and the fraction of the photon energy taken away by the positron.

The existing Geant $4 \gamma$-conversion models are appropriate for the simulation of EM showers, but are not accurate enough for a precise simulation of the high-performance $\gamma$-ray telescopes [18]. This is due to their sampling the polar angles of the two leptons independently and due to performing the decay in a plane containing the directions of the photon, of the electron and of the positron, without any allowance for a transverse out-ofplane recoil momentum. The class G4BetheHeitler5DModel [19-20] samples the full, 5D, Bethe-Heitler differential cross section, energy-momentum is strictly conserved and this model samples realistic correlations between the variables. 

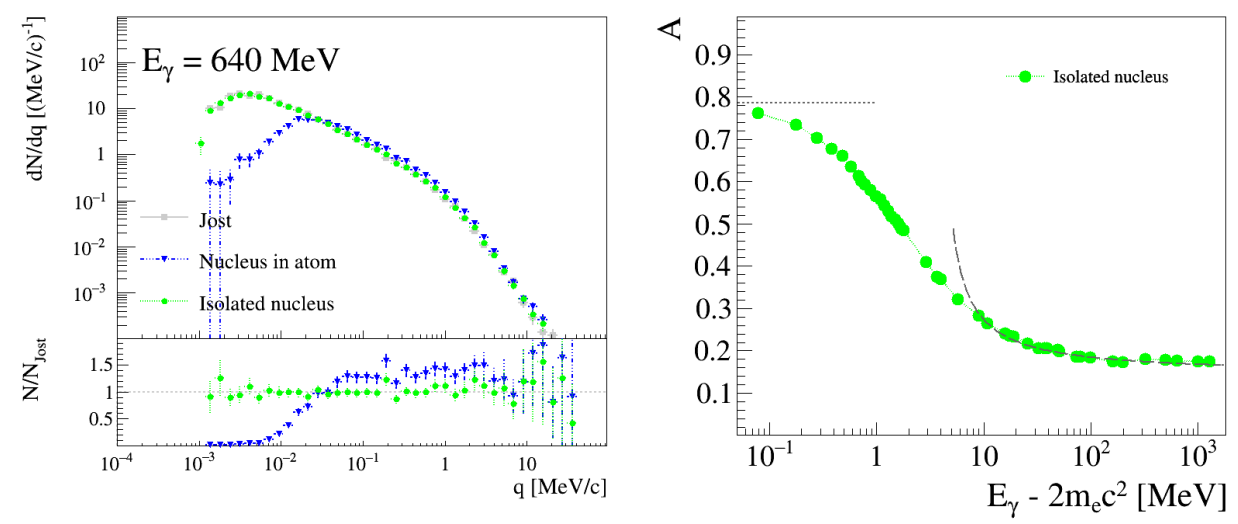

Fig. 2. Performance of the $5 \mathrm{D}$ gamma conversion model. Left: recoil momentum distribution compared to the analytical high-energy expression from [21]. For isolated targets, the simulation (bullets) matches nicely a distribution obtained from [21]. For nuclei inside an atom (triangles), the suppression of lowrecoil-momentum events is visible below a few $10^{-2} \mathrm{MeV} / \mathrm{c}$. The ratio plot is relative to [21]. Right: polarization asymmetry as a function of available energy, compared to published asymptotic expressions (dotted line - low energy from [22], dashed line - high energy from [23]).

The distribution of the magnitude of the recoil momentum is found (Fig.2 left and [19-20]) to be compatible with the analytical expression computed in the high-energy approximation [21]. The nuclear or triplet conversion of linearly polarized or non-polarized $\gamma$ rays on isolated or atomic targets is simulated. For linearly-polarized $\gamma$ rays, the polarization asymmetry is found (Fig.2 right and [19-20]) to be compatible with the known asymptotic expressions [22-23]. Because of 5D sampling, the new model is significantly slower than the default Bethe-Heitler model, so the 5D model is included only in the two EM physics constructors G4EmLivermorePhysics and G4EmLowEPPhysics.

\subsection{Livermore photoeffect model}

A revised Livermore model of the photoelectric effect is provided with Geant4 10.4. The total photoelectric and single shell cross-sections are based on the evaluated cross-section data from the EPICS2014 (Electron Photon Interaction Cross Sections) data library [24]. This updated library has been reported to be more accurate with respect to the previous version EPDL97 [25], previously used. Depending on the energy of the incident $\gamma$, the model provides cross-sections based either on the interpolation (linear or spline) of tabulated cross-sections or on the parameterisation of cross-sections data. The parameterisation over $\gamma$ energy $\mathrm{E}$ was obtained through two fits in two different energy ranges, with six parameters each, as follows:

$$
\sigma(E)=\frac{a_{1}}{E}+\frac{a_{2}}{E^{2}}+\frac{a_{3}}{E^{3}}+\frac{a_{4}}{E^{4}}+\frac{a_{5}}{E^{5}}+\frac{a_{6}}{E^{6}}
$$

The intervals ranges are set dynamically and they depend on the atomic number of the element and the corresponding K-shell binding energy.

These improvements allowed the reduction of the threshold for the parameterisation from $600 \mathrm{keV}$ to $5 \mathrm{keV}$, resulting in a measured speedup of about $20 \%$ for the final state generation sampling. In Fig. 3 the results of speedup of the updated Livermore model for different elements are shown. The simulations used $10^{6} \gamma$ with energy $0.4 \mathrm{MeV}$, and 20 repetitions for each element. 


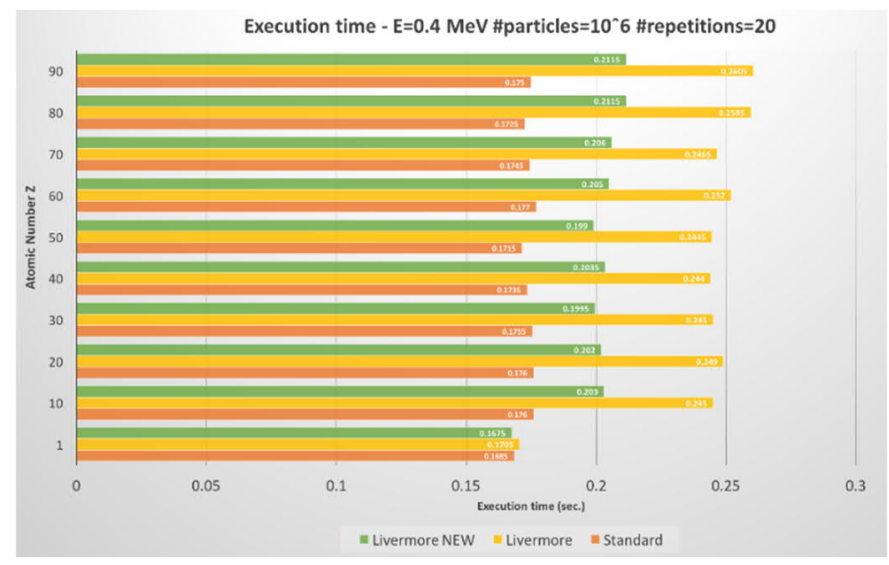

Fig. 3. Comparison of execution times between the updated Livermore model (green), the old Livermore (yellow) and the standard model (orange) as a function of the atomic number.

The angular distribution of the emitted $\mathrm{e}^{-}$follows the Sauter-Gavrila distribution [26] initially proposed for the K-shell. It is based on a new and optimised implementation (similarly to the model of Penelope 2014). This implementation is faster and gives a $4 \%$ to $20 \%$ speedup. The updated Livermore model has been tested and verified against other Geant 4 photo-electric models and the observed cross-sections have a maximum variation of $5 \%$ with respect to the Penelope cross section. For the majority of atoms and energy ranges cross sections agree within $1 \%$. Since Geant4 10.4, the new Livermore model is the default in all EM physics lists except Penelope.

\subsection{Gamma conversion to muon pair model}

The total cross section and the angular distribution of muons in Geant4 gamma conversion model is based on the multi-differential cross section [27] (G4GammaConversionToMuons class). For Geant4 10.4 we validated the total cross section of the process using direct integration of the Williams-Weizsacker differential cross section using Wolfram Mathematica 11.0 [28]. The obtained values for several targets agree within a few percent with the current parameterisation [27]. The model used for the final state generation was improved. The dependence of the angular distribution on the momentum transfer to the recoiling nucleus was taken into account, which slightly reduced scattering to the backward hemisphere. This update was inspired by the dark matter search experiment SHiP study on muon background and other projects in this area [8].

\subsection{Improved threshold for positron annihilation to $\mu^{+} \mu^{-}$}

The threshold positron energy in the laboratory system for the positron annihilation with atomic electrons into $\mu^{+} \mu^{-}$pair is $E_{\text {th }}=43.69 \mathrm{GeV}$. Taking into account that the electron is much lighter than the muon, the lowest order cross section can be written as

$$
\sigma=\frac{\pi r_{\mu}^{2}}{3} \varepsilon\left(1+\frac{\varepsilon}{2}\right) \sqrt{1-\varepsilon}
$$

where $r_{\mu}=r_{e} m_{e} / m_{\mu}$ is the classical muon radius, $m_{e}$ and $m_{\mu}$ are electron and muon masses, $r_{e}$ is the classical electron radius, $\varepsilon=\mathrm{E}_{\mathrm{th}} / \mathrm{E}_{\mathrm{lab}}$, where $\mathrm{E}_{\mathrm{lab}}$ is the total positron energy in the laboratory frame.

We now take into account the increase in cross section by the Sommerfeld-SchwingerSakharov (SSS) threshold Coulomb re-summation factor [29] 


$$
S(\beta)=\frac{X(\beta)}{1-e^{-X(\beta)}}, X(\beta)=\frac{\pi \alpha}{\beta \gamma}=\pi \alpha \frac{\sqrt{1-\beta^{2}}}{\beta},
$$

where $\alpha$ is the fine structure constant, $\beta$ and $\gamma$ are kinematic variables of muons in the centre of mass frame. The SSS-corrected cross section can be written at all energies as

$$
\sigma_{S S S}=\frac{\pi r_{\mu}^{2}}{3} \varepsilon\left(1+\frac{\varepsilon}{2}\right) \frac{\pi \alpha \sqrt{\varepsilon}}{1-e^{-\frac{\pi \alpha \sqrt{\varepsilon}}{\sqrt{1-\varepsilon}}}} .
$$

These expressions are used in the simulation of this process since Geant 4 10.5beta. The final factor in the expression is replaced by its asymptotic value of unity close to threshold to avoid numerical instabilities. The cross-section with and without the correction is shown in Fig.4. The noticeable increase of the cross section close to threshold by the SSS-factor is of practical interest for the low emittance production of muons, as proposed in [30].
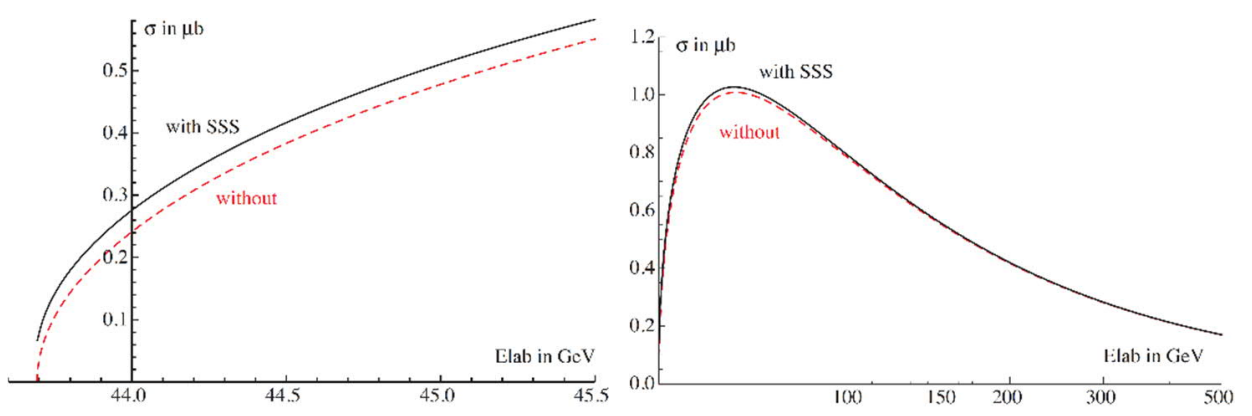

Fig. 5. Total cross section for $\mathrm{e}^{+} \mathrm{e}^{-} \rightarrow \mu^{+} \mu^{-}$as a function of the $\mathrm{e}^{+}$energy in the laboratory system. With (solid line) and without (dashed red line) SSS Coulomb re-summation factor, zoomed close to the threshold (left), and using a wider energy range (right).

\section{Updates to EM physics infrastructure}

Interfaces to material properties and density effect parameterisation for compounds were improved. Additional $\mathrm{C}++$ interfaces to $\mathrm{EM}$ parameters and corresponding UI commands were introduced. The method of automatic documentation for EM models, processes and physics list constructors has been developed. The web documentation for EM physics was reviewed and re-structured. Some of these developments will be described below.

\subsection{Geant4 material properties}

The interface for material properties defined in user code have been improved to allow significant speed-up, particularly in transport of optical photons. These properties are stored in a $\mathrm{C}++$ standard template library map. The keys for this map are now elements of an enumeration, replacing the char pointers used previously. The user still defines the material property vectors and constants in user code with AddProperty (const char* key, ...) or AddConstProperty(const char* key, ...). Here 'key' is, for example, 'RINDEX' for the refractive index. Corresponding physics process classes in the Geant4 distribution have been updated to retrieve the material property vectors and constants with GetProperty(G4int) and GetConstProperty(G4int) methods. The parameter is the enumerator, for example, $k R I N D E X$ for the refractive index.

In the user code, the material property vectors and constants may be retrieved using either the enumerator or the string label. Internally, a vector of string names is used to determine the integer corresponding to the string. In the case of a custom property (those not pre-defined 
in Geant4), an integer key is assigned automatically when the property is defined. In order to retrieve a custom property by index, the integer key may be first obtained using the methods GetPropertyIndex(G4String key) or GetConstPropertyIndex(G4String key).

\subsection{EM physics configuration}

In recent versions of Geant4, explicit EM model parameters were introduced [6-7]. Each EM physics list has a set of specific parameters which users may modify using User Interface (UI) commands or the $\mathrm{C}++$ interface. In Geant4 10.4 we extended the list of parameters and improve the visibility of the list in the output. In addition, we add extra methods to stream out model and process descriptions enabling automatic documentation generation for each EM configuration.

The default EM physics list (Opt0) is used in the majority of Geant4 Physics Lists. The Urban multiple scattering model is used for $\mathrm{e}^{ \pm}$below $100 \mathrm{MeV}$ [5]. In the Opt4 EM physics list the GS model of multiple scattering with Mott corrections enabled is used below 100 $\mathrm{MeV}$ instead of the Urban model. The Opt4 physics list uses the most accurate models for each process, as well as stricter configuration parameters for tracking of charged particles. The alternative EM physics list Opt3 may be considered as an intermediate variant between Opt0 and Opt4.

For testing purposes of novel calorimeters, we provide a configuration of electron scattering based on the GS model or on the SS model. In addition, the GS model with Mott corrections enabled is included in the Livermore and Penelope EM physics constructors. To improve usability, a new set of UI commands and corresponding $\mathrm{C}++$ interfaces have been added. In particular, the photo-absorption ionisation model may be enabled per detector region using the corresponding UI command.

Since version 10.4, three new EM physics constructors (G4EmDNAPhysics_optionX, where $\mathrm{X}=2,4$ or 6 ) are available. They allow a discrete simulation of particle interactions in liquid water, the main component of biological media, down to a few tens of eV. These constructors are developed in the context of the Geant4-DNA project [31-32]. The processes they include: ionization, electronic excitation, elastic scattering, vibrational excitation and molecular attachment for electrons. For protons, neutral hydrogen atoms, alpha particles and their charge states, the ionization, electronic excitation, elastic scattering, and charge exchange processes are available. These processes have been recently described in detail in [33-34] and the performance of these three physics constructors has been evaluated for nanoand micro-dosimetry simulations, notably through the usage of Geant4 extended examples [35-36]. A variety of their applications is described in [37].

\section{Summary}

Goudsmit-Sounderson model, which is the most accurate one for $\mathrm{e}^{ \pm}$transport, was improved in Geant4 10.4. The model may be recommended for a wide variety of simulation applications. Improvements in modelling of other processes, as well as in EM code infrastructure were introduced. Mott corrections were added to the WentzelVI model used for simulation of high energy scattering. In Geant 4 10.5beta the new 5D gamma conversion model was added. More effective handling of the material properties is provided, the interface to EM parameters is extended, and the set of EM physics list components is improved. We continue reviewing EM models in order to take into account various next-to-leading-order corrections to the cross sections and to the final state generation.

We acknowledge the support of the French National Research Agency (ANR-13-BS050002); Fermi Research Alliance, LLC under Contract No. DE-AC02-07CH11359 with the United States Department of Energy. 


\section{References}

1. J. Apostolakis et al., Radiation Physics and Chemistry 78, 859-873 (2009)

2. V. Ivanchenko et al., Progress in Nucl. Sci. and Technology 2, 898-903 (2011)

3. S. Agostinelli et al., Nucl. Instrum. Meth. A 506, 186-225 (2003)

4. J. Allison et al., IEEE Trans. Nucl. Sci. 53, 270-278 (2006)

5. J. Allison et al., Nucl. Instrum. Meth. A 835, 186-225 (2016)

6. A. Bagulya et al., IOP Conf. Series: J. Phys.: Conf. Series 898, 042032 (2017)

7. S. Incerti, V. Ivanchenko and M. Novak, JINST 13 C02054 (2018)

8. S. Incerti and V. Ivanchenko, EPJ Web of Conferences 142, 01016 (2017)

9. I. Kawrakow and A.F. Bielajew, Nucl. Instr. Meth B 134, 325-336 (1998)

10. S. Goudsmit and J. Saunderson, Phys. Rev. 57, 24 (1940)

11. N. Mott, Proc. Roy. Soc. 124, 425 (1929)

12. I. Kawrakow, Nucl. Instr. Meth. B 114, 307-326 (1996)

13. I. Kawrakow and A.F. Bielajew, Nucl. Instr. Meth B 142, 253-280 (1998)

14. G.J. Lockwood et al., Sandia report SAND79-0414, UC-34a (1987)

15. V.N. Ivanchenko et al., J. Phys.: Conf. Ser. 219, 032045 (2010)

16. G. Wentzel, Z. Phys. 40, 590 (1927)

17. H. Bethe and W. Heitler, Proceedings of the Royal Society of London A 146, 83 (1934)

18. P. Gros and D. Bernard, Astropart. Phys. 88, 60 (2017)

19. D. Bernard, Nucl. Instrum. Meth. A 899, 85 (2018)

20. I. Semeniouk and D. Bernard, http:://doi.org/10.1016/j.nima.2018.09.154

21. R. Jost, J.M. Luttinger, and M. Slotnick, Phys. Rev. 80, 189 (1950)

22. P. Gros and D. Bernard, Astropart. Phys. 88, 30 (2017)

23. V.F. Boldyshev and Y.P. Peresunko, Yad. Fiz. 14, 1027 (1971)

24. D.E. Cullen, EPICS2014, Doc. Series of the IAEA Nuclear Data Section (2014)

25. L.K. Dermott, E. Cullen, and J.H. Hubbell, EPDL97, UCRL-50400, 6, Rev. 5 (1997)

26. M. Gavrila, Phys. Rev. 113, 22 (1959)

27. H. Burkhardt et al., CERN-SL-2002-016-AP. CLIC-Note-511, CERN, Geneva (2002)

28. http://www.wolfram.com/mathematica/

29. S.J. Brodsky and R.F. Lebed, Phys. Rev. Lett. 102, 213401 (2009)

30. M. Boscolo et al., Phys. Rev. Accel. Beams 21, 061005 (2018)

31. http://geant4-dna.org

32. S. Incerti et al., Int. J. Model. Simul. Sci. Comput. 1, 157-178 (2010)

33. S. Incerti et al., Med. Phys. 37, 4692-4708 (2010)

34. M. A. Bernal et al., Phys. Med. 31, 861-874 (2015)

35. S. Incerti et al., Med. Phys. 45, e722-e739 (2018)

36. I. Kyriakou et al., Med. Phys. 42, 3870-3876 (2015)

37. S. Incerti et al., Phys. Med. 32, 1187-1200 (2016) 